Erratum

\title{
Erratum to "On type 1 representable lattices of dimension at most 4"
}

\author{
Grigore CăLugăreanu and Carolina Conţiu
}

ABStract. The main result in the paper mentioned above, that every lattice of length at most 4 and representable by commuting equivalence relations, is also representable by subgroups of an Abelian group, can be easily derived from known results due to B. Jónsson. Moreover, we correct some misleading statements.

It is well known (and trivial) that every lattice representable by subgroups of Abelian groups is also representable by commuting equivalence relations (often called type 1 representable). In the paper under discussion, our main result was Corollary 3.4, in which we stated that for modular lattices of dimension at most 4, the converse holds true. This result is actually an obvious consequence of Jónsson's [2, Theorem 3.1], which states that such a lattice admits an isometric embedding into the subspace lattice of some coordinatizable projective geometry.

The starting point of our approach was Jónsson's classification of modular lattices of length $\leq 4$. Nevertheless, we did not give due consideration to lattices containing simple complemented intervals of length 3 . What we did show is that any non-complemented finite 2-distributive modular lattice of length 4 admits an isometric embedding into the subgroup lattice of some finite abelian group having at most 3 cyclic summands, each of prime power order. This is the correct statement of our Theorem 3.3. Moreover, the proof of Theorem 3.3 required more cases to be taken into account, but these can be handled with the same methods.

Regarding the cases that were not addressed in our paper, if a lattice $L$ is arguesian of length $\leq 4$ containing a simple complemented length 3 interval $I$, then isometric embeddings into the subgroup lattice of some finite Abelian group seem to exist only under severe restrictions (C. Herrmann, private correspondence): $L$ has to be finite, $I$ a projective plane of order $p$ for $p$ a prime, and any length 2 interval of $L$ must be of size $\leq p+3$. The case where both

Presented by F. Wehrung.

Received February 19, 2013; accepted in final form April 16, 2013.

2010 Mathematics Subject Classification: Primary 06B15, Secondary 06C05.

Key words and phrases: type 1 representable lattice, Arguesian lattice, lattice representable by Abelian groups, dimension, length, socle, radical.

The online version of the original article can be found under doi:10.1007/s00012-012-0174-3.

Birkhäuser 
$1 / r$ and $s / 0$ are two simple height 3 complemented lattices glued over a height 2 interval is the most important one in Jónsson [2] with fundamental impact on the further development of modular lattice theory and its applications.

\section{Corrections.}

(a) In Theorem $1.1, \mathcal{A}, \mathcal{N}$, and $\mathcal{L}$ have to be replaced by $\mathcal{L}(\mathbb{Z}), \mathcal{N}($ rep $)$, and $\mathcal{T}_{1}$. Moreover, given the fact that the class $\mathcal{L}$, of all linear lattices, is (by definition) closed under sublattices, we have $\mathcal{L}=\mathcal{T}_{1}$.

(b) In Remark 2.1, one has to consider the 3-element chain, which is not complemented. Indeed, this lattice is isomorphic to $L\left(\mathbf{Z}_{p^{2}}\right)$ for any prime number $p$. The sentence following this Remark should read: "Restricted to the class of lattices $L$ of length two, the classes $\mathcal{L}(\mathbb{Z})$, $\mathcal{N}$ (rep), and $\mathcal{T}_{1}$ coincide; moreover, if also $|L| \geq 5$, then one has $L \in \mathcal{A}$ if and only if $L \in \mathcal{N}$, and the latter is equivalent to $L \in \mathcal{T}_{1}$ in case (and only in this case) that $|L|=p+3$ for $p$ a prime."

(c) The last sentence, page 137, line -2 should read: "As for the upper left corner of Figure $4 \ldots$.

(d) Theorem 3.3 and Corollary 3.4 are obvious consequences of [2, Theorem 3.1].

(e) Our paper ended with an open question, whether the equality $\mathcal{N}=\mathcal{T}_{1}$ holds for (modular) lattices of length at most 4 . This is answered in the negative in $[3]$.

Acknowledgement. Thanks are due to Christian Herrmann for his kind guidance in writing this corrigenda.

\section{REFERENCES}

[1] Călugăreanu G., Conţiu C.: Type 1 representable lattices of dimension at most 4. Algebra Universalis 67, 131-139 (2012)

[2] Jónsson, B.: Arguesian lattices of dimension $n \leq 4$. Math. Scand. 7, 131-145 (1959)

[3] Herrmann C.: A review of some of Bjarni Jónsson's results on representation of Arguesian lattices. Algebra Universalis 70, 163-174 (2013)

\section{Grigore Călugăreanu and Carolina Conţiu}

Faculty of Mathematics and Computer Science, Babeş-Bolyai University, Cluj-Napoca, Romania 400084

e-mail, Grigore Călugăreanu: calu@math.ubbcluj.ro, calug2001@yahoo.com

e-mail, Carolina Conţiu: carolinacontiu@yahoo.com

URL, Grigore Călugăreanu: http://math.ubbcluj.ro/ calu 